\title{
Mirrors with regular hexagonal segments
}

\author{
Dario Amodei and Stephen Padin
}

\begin{abstract}
The point-spread function and emissivity are calculated for a mirror made from regular hexagonal segments of just a few different sizes. A mirror of this type has many similar segments, which is an advantage for manufacturing, and for an $\sim f / 1$ mirror with $\geq 1000$ segments and $\geq 4$ sizes of regular hexagons the increase in intersegment gap area is negligible. This result raises the possibility of making a mirror from very large numbers of identical small segments that are warped to the required figure. (C) 2003 Optical Society of America

OCIS codes: $350.1260,220.4880,110.6770$.
\end{abstract}

\section{Introduction}

A segmented mirror must have intersegment gaps to accommodate segment manufacturing tolerances, handling, and gravitational and thermal deformations of the mirror cell. For small segments, gravitational deformations limit the gap width to $w>$ $\xi d / D$, where $\xi$ is the gravitational deflection of the cell, $d$ is the segment diameter, and $D$ is the mirror diameter. For large segments, tolerances and handling may be more important in setting the gap width. Intersegment gaps are difficult to mask at a cold image, so they increase the emissivity of the mirror. Gaps also cause point-spread function (PSF) sidelobes that can severely limit high-contrast measurements. ${ }^{1}$ An obvious approach to minimizing these effects is to make the gap area as small as possible. In addition, if the projection of the segments in the aperture plane is an array of regular hexagons, the PSF will have a hexagonal array of sidelobes, but there will be clean regions very close to the PSF core. This scheme requires irregular hexagonal segments, and each size and shape of segment is repeated only six times in the mirror. ${ }^{2}$ The approach is reasonable for a mirror with a few hundred segments, but as the number of segments increases, cost savings through high-quantity production favor designs with many similar segments. Tiling a mir-

The authors are with the California Institute of Technology, Mail Stop 105-24, 1200 East California Boulevard, Pasadena, California 91125. D. Amodei's e-mail address is dario@caltech.edu; S. Padin's is spadin@caltech.edu.

Received 25 October 2002; revised manuscript received 25 February 2003.

0003-6935/03/255130-06\$15.00/0

(C) 2003 Optical Society of America ror with regular hexagons is an attractive option because it reduces the tooling cost, but hexagons of a few different sizes are necessary to avoid a large gap area. This idea can be extended to more radical manufacturing approaches, such as making a mirror from a few batches each of many, identical, small, thin, regular hexagonal segments that are mechanically warped ${ }^{3}$ into the appropriate figure for their location in the mirror.

Design issues for a mirror with regular hexagonal segments are explored in the following sections. In Section 2 we calculate the gap area for a mirror tiled with regular hexagons whose diameters decrease with distance from the optical axis. The analysis is expanded in Section 3 to include hexagons of just a few different sizes, and the PSF for a mirror of this type is calculated in Section 4.

\section{Tiling with Regular Hexagons}

When an axially symmetric curved surface is tiled with regular hexagons, the gap area can be minimized by varying the diameters of the hexagons with distance from the axis. Starting with a planar array of regular hexagons, we must apply a compression function, $v(r)=\sum_{n=1}^{\infty} c_{n} r^{n}$, and then fold the plane onto the curved surface. The combination of compression and folding must preserve the shapes (but not the sizes) of the hexagons; i.e., the distortions in the radial and azimuthal directions must be the same. Applying $v(r)$ causes a compression $v^{\prime}(r)$ in the radial direction and a compression $v(r) / r$ in the azimuthal direction. Folding maps a circle of radius $r$ in the plane to a circle of radius $\rho(r)$ on the surface (see Fig. 1), resulting in an additional azimuthal compression $\rho(r) / r$. The total azimuthal compression is 


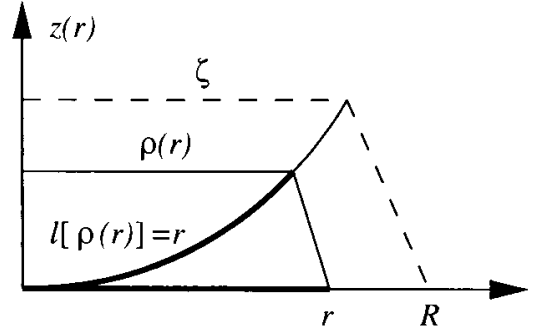

Fig. 1. Folding a plane onto an axially symmetric curved surface.

$[v(r) / r] \rho[v(r)] / v(r)$, so for equal radial and azimuthal compressions,

$$
v^{\prime}(r)=\frac{\rho[v(r)]}{r} .
$$

The distance along the surface from the origin to a point at radius $\rho$ is $l[\rho(r)]=r$ (see Fig. 1), and substituting $\rho$ from Eq. (1) gives

$$
l\left[r v^{\prime}(r)\right]=v(r) .
$$

The function $l(\rho)$ is odd, with $l^{\prime}(0)=1$ [see Eq. (6)], so we can expand $l(\rho)$ as

$$
l(\rho)=\sum_{n=0}^{\infty} a_{2 n+1} \rho^{2 n+1},
$$

with $a_{1}=1$. Solving Eq. (2) as a power series then gives

$$
v(r)=r-\frac{1}{2} a_{3} r^{3}+\frac{1}{8}\left(9 a_{3}^{2}-2 a_{5}\right) r^{5}+\ldots .
$$

We want an expression for $v(r)$ in terms of the profile of the curved surface, $z(\rho)$. For an axially symmetric surface, ${ }^{4}$

$$
z(\rho)=\sum_{n=1}^{\infty} b_{2 n} \rho^{2 n}
$$

so

$$
\begin{aligned}
l(\rho) & =\int_{0}^{\rho}\left[1+z^{\prime}(t)^{2}\right]^{1 / 2} \mathrm{~d} t \\
& =\rho+\frac{2}{3} b_{2}{ }^{2} \rho^{3}+\frac{2}{5}\left(4 b_{2} b_{4}-b_{2}{ }^{4}\right) \rho^{5}+\ldots \\
v(r) & =r-\frac{1}{3} b_{2}{ }^{2} r^{3}+\frac{1}{5}\left(3 b_{2}{ }^{4}-2 b_{2} b_{4}\right) r^{5}+\ldots
\end{aligned}
$$

For a paraboloidal mirror, $b_{2}=1 / 2 k$, where $k$ is the radius of curvature, and $b_{4}=0$; hence

$$
\begin{gathered}
v(r) \approx r-\frac{1}{12 k^{2}} r^{3}+\frac{3}{80 k^{4}} r^{5}, \\
v^{\prime}(r) \approx 1-\frac{1}{4 k^{2}} r^{2}+\frac{3}{16 k^{4}} r^{4} .
\end{gathered}
$$

The $r^{6}$ term in $v^{\prime}(r)$ is approximately $-0.1927 r^{6} / k^{6}$ and is negligible for $r / k \ll 1$, i.e., for focal ratios larger than $\sim f / 0.5$.
Table 1. Segment Diameter and Gap Width for a 30-m $f / 0.75$ Mirror with 1-m Segments

\begin{tabular}{llcl}
\hline \multicolumn{1}{c}{ Parameter } & Units & Eqs. (10) \& (12) & Simulation \\
\hline Minimum diameter & $\mathrm{m}$ & 0.973653 & 0.973263 \\
Average diameter & $\mathrm{m}$ & 0.986206 & 0.986102 \\
Average gap & $\mathrm{mm}$ & 0.619 & 0.580 \\
\hline
\end{tabular}

Now we can start drawing hexagons. If $d$ is the diameter of the hexagons in the original planar array, regular hexagonal segments of diameter $h_{\mathrm{av}}(r)=$ $d v^{\prime}(r)$ will overlap adjacent segments at larger $r$ (but the average gap width around a segment will be close to zero). Hexagons of diameter $d v^{\prime}(r+d / 2)$ will fit with no overlaps because only the outermost parts of a hexagon must be compressed by $v^{\prime}(r+d / 2)$, but we can clearly tile with somewhat larger segments, i.e., with a diameter in the range of $d v^{\prime}(r+d / 2)$ to $d v^{\prime}(r)$. The center of this range gives a rough estimate of the maximum segment diameter,

$$
\begin{aligned}
h(r) & =d v^{\prime}\left(r+\frac{d}{4}\right) \\
& \approx d\left[1-\frac{1}{4 k^{2}}\left(r+\frac{d}{4}\right)^{2}+\frac{3}{16 k^{4}}\left(r+\frac{d}{4}\right)^{4}\right],
\end{aligned}
$$

and the corresponding gap width, expressed as a change in segment diameter, is

$\Delta h(r)=h_{\mathrm{av}}(r)-h(r)=d\left[v^{\prime}(r)-v^{\prime}\left(r+\frac{d}{4}\right)\right] \approx \frac{d^{2} r}{8 k^{2}}$.

The average gap width over the entire mirror is then

$$
\langle\Delta h(r)\rangle \approx \frac{1}{\pi R^{2}} \int_{0}^{R} \frac{d^{2} r}{8 k^{2}} 2 \pi r \mathrm{~d} r=\frac{2}{3} \Delta h(R),
$$

where $R \approx \zeta\left(1+\zeta^{2} / 6 k^{2}\right)$ is the radius of the mirror measured along the surface and $\zeta$ is the radius measured perpendicular to the optical axis (see Fig. 1). Table 1 compares estimates of the segment diameter and average gap width from Eqs. (10) and (12) with results from a computer simulation of tiling a $30-\mathrm{m}$ f/0.75 mirror with 1-m segments. The simulation calculates the vertices of the irregular hexagonal regions produced by compressing and folding a flat sheet of regular hexagons. It then inscribes the largest possible regular hexagon in each region, adjusting position and rotation in the plane of the hexagon to maximize the diameter. Figure 2 compares Eq. (11) with the simulation results for $\Delta h(r)$. In general, the simulation gives slightly larger segments, and hence smaller gaps, than the simple model of Eqs. (10) and (11).

\section{Batches of Segments}

To gain a significant manufacturing advantage, we want to tile the mirror with regular hexagons of just a few different diameters. Each batch of hexagons 


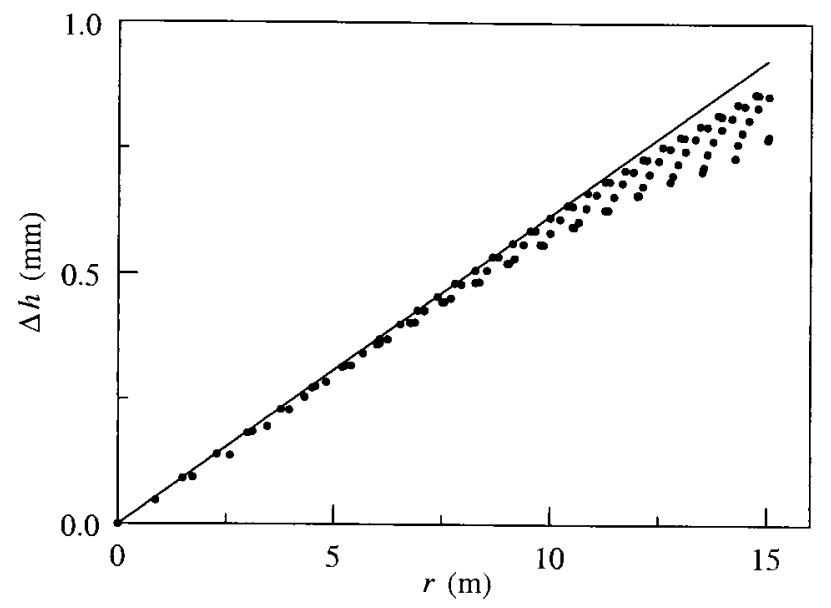

Fig. 2. Gap width predicted by Eq. (11) and results from a computer simulation (points) for a $30-\mathrm{m} f / 0.75$ mirror tiled with $1-\mathrm{m}$ diameter regular hexagons.

will occupy an annulus, and we must choose the boundaries $\left(r_{0}=0, r_{1}, r_{2}, \ldots, r_{n}=R\right)$ to minimize the gap area. The difference between the actual diameter of a hexagon in the region $r_{i}<r<r_{i+1}$ and the diameter permitted by Eq. (10) is

$$
\delta(r) \approx \frac{d}{4 k^{2}}\left(r_{i+1}^{2}-r^{2}\right),
$$

where $\delta\left(r_{i}\right)=0$ and we have ignored terms in $r^{4}$ and higher. (This results in a small error in the choice of boundaries.) The average difference over the mirror is

$$
\begin{aligned}
\langle\delta(r)\rangle & =\frac{1}{\pi R^{2}} \sum_{i=0}^{n-1} \int_{0}^{R} \delta(r) 2 \pi r \mathrm{~d} r \\
& \approx \frac{d}{8 k^{2} R^{2}} \sum_{i=0}^{n-1}\left(r_{i+1}{ }^{2}-r_{i}^{2}\right)^{2},
\end{aligned}
$$

which is minimized by choosing $r_{i}{ }^{2}=i R^{2} / n$, so each batch occupies the same area on the surface of the mirror. The diameter of a hexagon in the region $r_{i}<$ $r<r_{i+1}$ is then

$$
h\left(r_{i}\right) \approx d\left[1-\left(\frac{i}{n}\right)^{1 / 2} \frac{R d}{8 k^{2}}-\frac{i R^{2}}{4 n k^{2}}+\frac{3 i^{2} R^{4}}{16 n^{2} k^{4}}\right] .
$$

Now we can calculate the gap width and estimate the emissivity of the mirror. The average diameter of the batched regular hexagons is

$$
\begin{aligned}
\left\langle h\left(r_{i}\right)\right\rangle= & \frac{1}{n} \sum_{i=1}^{n} h\left(r_{i}\right) \\
\approx & d\left[1-\left(\frac{n+1}{2 n}\right)^{1 / 2} \frac{R d}{8 k^{2}}-\frac{n+1}{2 n} \frac{R^{2}}{4 k^{2}}\right. \\
& \left.+\frac{(n+1)(2 n+1)}{6 n^{2}} \frac{3 R^{4}}{16 k^{4}}\right],
\end{aligned}
$$

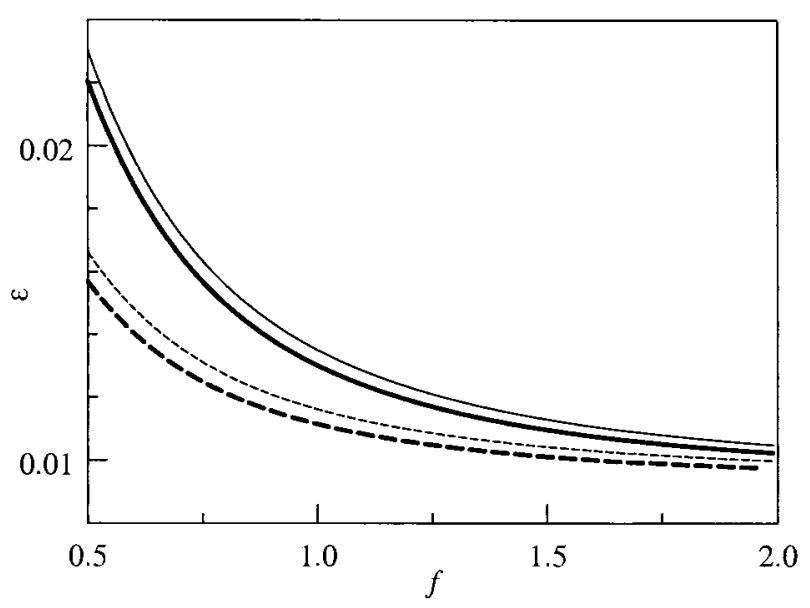

Fig. 3. Emissivity as a function of focal ratio [Eq. (19)] for mirrors with four (solid curves) and eight (dashed curves) batches of regular hexagonal segments with $d / k=1 / 90$ (light curves) and $d / k=$ $1 / 900$ (bold curves). The nominal gap width is $s / d=0.004$.

where we have used the rms value of $(i / n)^{1 / 2}$ as an (over)estimate of $(1 / n) \sum_{i=1}^{n}(i / n)^{1 / 2}$. The average diameter of the irregular hexagonal regions occupied by the segments is

$$
\left\langle h_{\mathrm{av}}\right\rangle=\frac{1}{\pi R^{2}} \int_{0}^{R} d v^{\prime}(r) 2 \pi r \mathrm{~d} r \approx d\left[1-\frac{R^{2}}{8 k^{2}}+\frac{R^{4}}{16 k^{4}}\right],
$$

so the gap width, expressed as a change in segment diameter, is $\Delta h_{\text {batch }}=\left\langle h_{\text {av }}\right\rangle-\left\langle h\left(r_{i}\right)\right\rangle$. We must also include a nominal spacing between hexagons to allow for gravitational and thermal deformations of the mirror cell, manufacturing tolerances, and segment handling. If the nominal spacing is $s$, the average diameter of the batched hexagons is reduced by $2 s$ / $\sqrt{3}$, so

$$
\begin{aligned}
\Delta h_{\mathrm{batch}} & =\left\langle h_{\mathrm{av}}\right\rangle-\left\langle h\left(r_{i}\right)\right\rangle+\frac{2 s}{\sqrt{3}} \\
& \approx\left(\frac{n+1}{2 n}\right)^{1 / 2} \frac{R d^{2}}{8 k^{2}}+\frac{R^{2} d}{8 n k^{2}}-\frac{3 R^{4} d}{32 n k^{4}}+\frac{2 s}{\sqrt{3}} .
\end{aligned}
$$

The fraction of the mirror area in the gaps is $2 \Delta h / d$, so the emissivity is

$$
\varepsilon \approx\left(\frac{n+1}{2 n}\right)^{1 / 2} \frac{1}{16 f} \frac{d}{k}+\frac{1}{64 n f^{2}}-\frac{3}{4096 n f^{4}}+\frac{4 s}{d \sqrt{3}},
$$

where $f=k / 4 \zeta$ is the focal ratio. Figure 3 shows the emissivity for several segment diameters and nominal gap widths. If we allow the emissivity due to the 


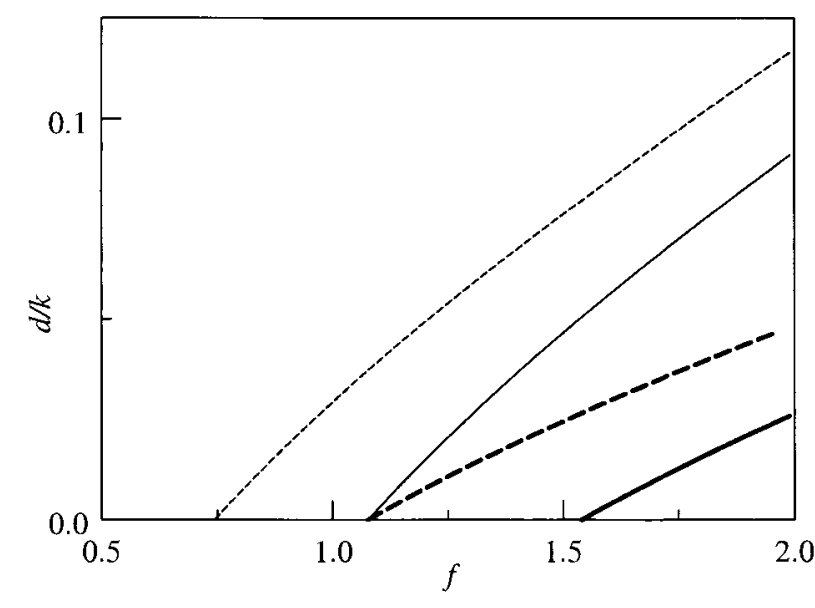

Fig. 4. Maximum segment diameter as a function of focal ratio [Eq. (20)] for a 35\% increase in emissivity due to tiling with four (solid curves) and eight (dashed curves) batches of regular hexagons with $s / d=0.004$ (light curves) and $s / d=0.002$ (bold curves).

gaps to increase by a factor $\eta$ because of batching, the segment diameter is limited to

$$
\frac{d}{k} \leq\left(\frac{2 n}{n+1}\right)^{1 / 2}\left[\frac{64 f s(\eta-1)}{d \sqrt{3}}-\frac{1}{4 f n}\left(1-\frac{3}{64 f^{2}}\right)\right],
$$

which is shown in Fig. 4 for $\eta=1.35$. As an example, the 30-m f/1.5 California Extremely Large Telescope $^{5}$ has 1 -m diameter segments $(d / k=1 / 90)$ with 4-mm nominal gaps (2-mm physical gaps and 1-mm safety bevels, giving $s / d=4 \times 10^{-3}$ ), so tiling with four batches of regular hexagons would increase the emissivity due to gaps from $0.92 \%$ to $1.13 \%$. This is a small effect compared with 3-6\% emissivity for the three reflections in the telescope.

\section{Point-Spread Function}

In a mirror tiled with regular hexagons, the segment diameter decreases away from the axis. In addition, the projection of the segments onto the aperture plane causes a radial contraction that increases away from the axis. These effects smear the PSF sidelobes and can limit the sensitivity of high-contrast measurements near the PSF core.

Calculating the PSF for a mirror with hexagonal segments is fairly straightforward once the segment vertices are known because the projection of each segment in the aperture plane is a linear transformation of a regular hexagon. This can be written as the sum of three linear transformations of a square. If $H(\mathbf{x})$ is a hexagon centered at the origin with $H(\mathbf{x})$ $=1$ inside the hexagon and $H(\mathbf{x})=0$ outside, and $S(\mathbf{x})$ is a unit square with its lower left corner at the origin, then

$$
H(\mathbf{x})=\sum_{i j=12,23,31} S\left(\mathbf{A}_{i j}{ }^{-1} \mathbf{x}\right),
$$

where $\mathbf{A}_{i j} \mathbf{e}_{1}=\mathbf{x}_{i}$ and $\mathbf{A}_{i j} \mathbf{e}_{2}=\mathbf{x}_{j} ; \mathbf{x}_{1}, \mathbf{x}_{2}, \mathbf{x}_{3}$ are vectors from the center to three alternating vertices of the hexagon; and $\mathbf{e}_{1}=(1,0)$ and $\mathbf{e}_{2}=(0,1)$ are the standard basis vectors. For hexagons not centered at the origin, there is a translation factor that we will add later. The Fourier transform (FT) of $H(\mathbf{x})$ is

$$
\phi(\mathbf{u})=\sum_{i j=12,23,31} \operatorname{FT}\left[S\left(\mathbf{A}_{i j}{ }^{-1} \mathbf{x}\right)\right]=\sum \operatorname{det}\left(\mathbf{A}_{i j}\right) \psi\left(\mathbf{A}_{i j}{ }^{t} \mathbf{u}\right),
$$

where we have used $\operatorname{FT}\left[S\left(\mathbf{A}^{-1} \mathbf{x}\right)\right]=\operatorname{det}(\mathbf{A}) \psi\left(\mathbf{A}^{t} \mathbf{u}\right)$, where $\psi(\mathbf{u})=$ FT $[S(\mathbf{x})]$ (see Appendix A). The Fourier transform of $S(\mathbf{x})$ is a separable product, $\psi(\mathbf{u})=$ $F\left(\mathbf{u} \cdot \mathbf{e}_{1}\right) F\left(\mathbf{u} \cdot \mathbf{e}_{2}\right)$, where $F(u)=(1 / u)[\sin u-i(1-\cos$ $u)]$ for a square of unit area, ${ }^{6}$ so

$$
\begin{aligned}
\phi(\mathbf{u}) & =\sum_{i j=12,23,31} \operatorname{det}\left(\mathbf{A}_{i j}\right) F\left(\mathbf{A}_{i j}^{t} \mathbf{u} \cdot \mathbf{e}_{1}\right) F\left(\mathbf{A}_{i j}^{t} \mathbf{u} \cdot \mathbf{e}_{2}\right) \\
& =\sum \operatorname{det}\left(\mathbf{A}_{i j}\right) F\left(\mathbf{A}_{i j} \mathbf{e}_{1} \cdot \mathbf{u}\right) F\left(\mathbf{A}_{i j} \mathbf{e}_{2} \cdot \mathbf{u}\right) \\
& =\sum \operatorname{det}\left(\mathbf{A}_{i j}\right) F\left(\mathbf{x}_{i} \cdot \mathbf{u}\right) F\left(\mathbf{x}_{j} \cdot \mathbf{u}\right) .
\end{aligned}
$$

Each determinant in Eq. (23) represents the area of a transformed square, so $\operatorname{det}\left(\mathbf{A}_{12}\right)=\operatorname{det}\left(\mathbf{A}_{23}\right)=$ $\operatorname{det}\left(\mathbf{A}_{31}\right)$. The area includes three scale factors: $\sqrt{3}$ for converting a unit square into a parallelogram that is part of a unit hexagon; $[h(\rho) / 2]^{2}$ for scaling the hexagon to edge length $h(\rho) / 2$; and $\left[1+z^{\prime}(\rho)^{2}\right]^{-1 / 2}$, which is the cosine of the radial tilt of the surface at radius $\rho$. For a hexagon centered at $\rho$, we must also include the phase factor $\exp (-i \mathbf{u} \cdot \boldsymbol{\rho})$, so

$$
\begin{aligned}
\phi(\mathbf{u})= & {\left[\frac{h(\rho)}{2}\right]^{2}\left[\frac{3}{1+z^{\prime}(\rho)^{2}}\right]^{1 / 2} \exp (-i \mathbf{u} \cdot \boldsymbol{\rho}) } \\
& \times \sum_{i j=12,23,31} F\left(\mathbf{x}_{i} \cdot \mathbf{u}\right) F\left(\mathbf{x}_{j} \cdot \mathbf{u}\right) \\
= & {\left[\frac{h(\rho)}{2}\right]^{2}\left[\frac{3}{1+z^{\prime}(\rho)^{2}}\right]^{1 / 2} } \\
& \times \frac{\exp (-i \mathbf{u} \cdot \boldsymbol{\rho})}{\left(\mathbf{x}_{1} \cdot \mathbf{u}\right)\left(\mathbf{x}_{2} \cdot \mathbf{u}\right)\left(\mathbf{x}_{3} \cdot \mathbf{u}\right)} \\
& \times \sum_{i=1}^{3}\left(\mathbf{x}_{i} \cdot \mathbf{u}\right) \cos \left(\mathbf{x}_{i} \cdot \mathbf{u}\right) .
\end{aligned}
$$

The PSF for the mirror is the sum of $\phi(\mathbf{u})$ for all the segments. This is shown in Fig. 5(a) for a $30-\mathrm{m}$ f/0.75 mirror tiled with four batches of 1-m diameter regular hexagons with a nominal gap width of $4 \mathrm{~mm}$. In this case the mirror is spherical, which is computationally a little easier than a paraboloid. For comparison, Fig. 5(b) shows the PSF for segments that are projected as 1-m regular hexagons in the aperture plane. The inner sidelobes are higher for the batched regular hexagons, but the outer sidelobes are lower because they are smeared by variations in the projected segment diameter and intersegment gap width. If the focal ratio is increased to $f / 1.5$, the PSF plots become essentially identical. For constant $s / d$, smaller segments give lower PSF sidelobes because the gap width due to batching is smaller. 


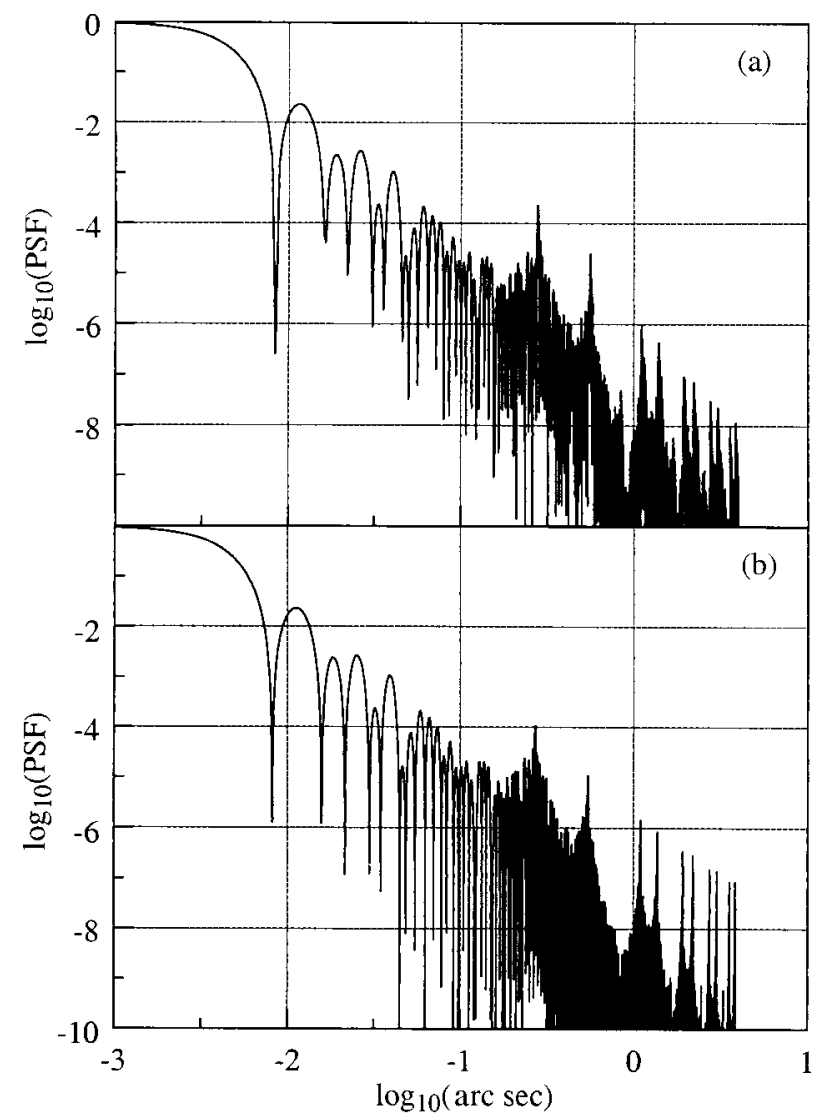

Fig. 5. (a) PSF for a $30-\mathrm{m} f / 0.75$ mirror tiled with four batches of $1-\mathrm{m}$ diameter regular hexagons with $4-\mathrm{mm}$ nominal gaps. The plot is taken through the sidelobes closest to the PSF core, and the wavelength is $1 \mu \mathrm{m}$. (b) PSF for a 30-m mirror with segments that are projected in the aperture plane as 1-m diameter regular hexagons with 4-mm gaps.

This allows the construction of faster mirrors, as well as mirrors with lower PSF sidelobes. Figure 6 shows the PSF for a 30-m f/0.75 mirror with four batches of $0.1-\mathrm{m}$ diameter regular hexagons. The

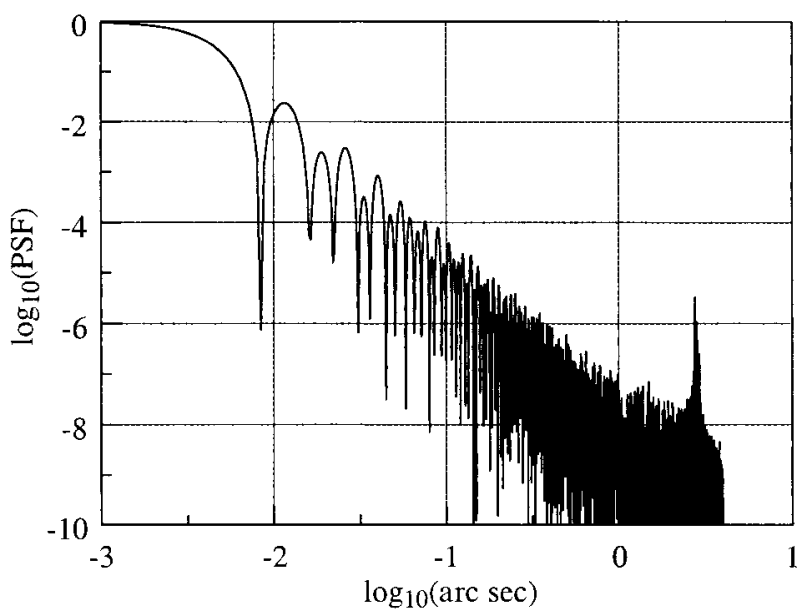

Fig. 6. PSF for a $30-\mathrm{m} f / 0.75$ mirror tiled with four batches of $0.1-\mathrm{m}$ diameter regular hexagons with $0.4-\mathrm{mm}$ nominal gaps. The wavelength is $1 \mu \mathrm{m}$. corresponding PSF for segments that are projected as regular hexagons in the aperture plane is almost identical. Figures 5 and 6 illustrate an important advantage of very small segments for high-contrast observations of extrasolar planets, where a low sidelobe level $\sim 0.1-1$ arc sec from the PSF core is critical. ${ }^{7}$ With 1-m segments, the gaps cause significant sidelobe structure to within a few tenths of an arc second of the PSF core, but with 0.1-m segments, the first sidelobe due to intersegment gaps is at a few arc seconds.

\section{Discussion}

Mirrors with more than $\sim 1000$ segments can be tiled with regular hexagons without compromising the emissivity and PSF. The approach could reduce tooling costs for existing large telescope designs and is very important for highly segmented mirrors because high-quantity production of identical segments can significantly reduce the cost. Small, thin, mechanically warped, regular hexagonal segments could make a cost-effective off-axis telescope possible. This would avoid secondary support diffraction effects, resulting in a very clean PSF. Since small segments have low mass, gravitational deflections in the mirror cell are reduced and the fractional gap width can be made smaller. Small segments also require small safety bevels and small handling gaps, so a highly segmented mirror made with regular hexagons can achieve very low emissivity.

\section{Appendix A: Fourier Transform of a Two-Dimensional Linearly Mapped Function}

This is the Similarity Theorem ${ }^{6}$ for a linear map $L$ : $\mathbf{R}^{2} \rightarrow \mathbf{R}^{2}$. Let $\mathbf{A}\left(x^{\prime}, y^{\prime}\right)=(x, y)$, where

$$
\mathbf{A}=\left(\begin{array}{ll}
a & c \\
b & d
\end{array}\right)
$$

and let $f(x, y)$ be a complex-valued function. The Fourier transform of $f\left[\mathbf{A}^{-1}(x, y)\right]$ is

$$
\begin{aligned}
\mathrm{FT}\left\{f\left[\mathbf{A}^{-1}(x, y)\right]\right\}= & \int_{-\infty}^{\infty} \int_{-\infty}^{\infty} f\left[\mathbf{A}^{-1}(x, y)\right] \\
& \times \exp (-i u x) \exp (-i v y) \mathrm{d} x \mathrm{~d} y \\
= & \int_{-\infty}^{\infty} \int_{-\infty}^{\infty} f\left(x^{\prime}, y^{\prime}\right) \exp \left[-i u\left(a x^{\prime}\right.\right. \\
& \left.\left.+c y^{\prime}\right)\right] \exp \left[-i v\left(b x^{\prime}+d y^{\prime}\right)\right] \mathrm{d} x \mathrm{~d} y \\
= & \int_{-\infty}^{\infty} \int_{-\infty}^{\infty} f\left(x^{\prime}, y^{\prime}\right) \exp \left[-i x^{\prime}(u a\right. \\
& +v b)] \exp \left[-i y^{\prime}(u c+v d)\right] \\
& \times|J(\mathbf{A})| \mathrm{d} x^{\prime} \mathrm{d} y^{\prime} \\
= & \operatorname{det}(\mathbf{A}) F\left[\mathbf{A}^{t}(u, v)\right],
\end{aligned}
$$

where $F(u, v)=\operatorname{FT}[f(x, y)]$. 
This work was supported by the Brewer Prize Undergraduate Fellowship awarded to Dario Amodei.

\section{References}

1. J. R. Kuhn, G. Moretto, R. Racine, F. Roddier, and R. Coulter, "Concepts for a large-aperture high dynamic range telescope," Astron. Soc. Pac. 113, 1486-1510 (2001).

2. J. E. Nelson, T. S. Mast, and S. M. Faber, eds., "The Design of the Keck Observatory and Telescope," Keck Observatory Report 90 (Keck Observatory, Kamuela, Hawaii, 1985).

3. T. S. Mast and J. E. Nelson, "The fabrication of large optical surfaces using a combination of polishing and mirror bending," in Advanced Technology Optical Telescopes IV, L. D. Barr, ed., Proc. SPIE 1236, 670-681 (1990).

4. R. N. Wilson, Reflecting Telescope Optics I (Springer-Verlag, Berlin, Germany, 1996), Chap. 2, p. 27.

5. J. E. Nelson and T. S. Mast, eds., "Conceptual design for a thirty-meter telescope," California Extremely Large Telescope Report 34 (University of California, Santa Cruz, 2002).

6. R. N. Bracewell, The Fourier Transform and Its Applications (McGraw-Hill, New York, 1986), Chap. 6, p. 245.

7. N. Woolf and J. R. Angel, "Astronomical searches for Earth-like planets and signs of life," Annu. Rev. Astron. Astrophys. 36, 507-537 (1998). 